\title{
Holism and structural realism
}

\author{
Michael Esfeld \& Vincent Lam \\ University of Lausanne, Department of Philosophy \\ CH-1015 Lausanne, Switzerland \\ Michael-Andreas.Esfeld@unil.ch, Vincent.Lam@unil.ch
}

\begin{abstract}
(published in Robrecht Vanderbeeken and Bart D'Hooghe (eds.): Worldviews, science and us. Studies of analytical metaphysics. A selection of topics from a methodological perspective.
\end{abstract}

Singapore: World Scientific 2010, pp. 10-31)

\begin{abstract}
We first introduce structural realism as a position in the metaphysics of science, pointing out the way in which this position replaces intrinsic properties with relations so that it amounts to a holistic in contrast to an atomistic metaphysics. We argue in favour of a moderate version of structural realism that puts objects and relations on the same ontological footing and assess the general philosophical arguments for this position. The second section shows how structural realism gains support from quantum physics. The third section explains how structural realism can be applied to the metaphysics of space-time.
\end{abstract}

\section{Structural realism as a metaphysical position}

Structural realism in the metaphysics of science is a sort of a holism in contrast to an atomism. To bring out that contrast, consider David Lewis' thesis of Humean supervenience; one can regard that thesis as the paradigmatic conception of a philosophical atomism in current mainstream analytical metaphysics:

Humean supervenience is named in honor of the greater denier of necessary connections. It is the doctrine that all there is to the world is a vast mosaic of local matters of particular fact, just one little thing and then another. (...) We have geometry: a system of external relations of spatio-temporal distance between points. Maybe points of spacetime itself, maybe point-sized bits of matter or aether or fields, maybe both. And at those points we have local qualities: perfectly natural intrinsic properties which need nothing bigger than a point at which to be instantiated. For short: we have an arrangement of qualities. And that is all. (Lewis (1986), ix-x) According to Lewis, thus, the only irreducible relations are the ones of spatio-temporal distance between points. The fundamental physical 11 properties are instantiated at those points. One may think of mass, energy, momentum, charge, spin among others as candidates for fundamental physical properties. It is of no importance for this position whether (a) the space-time points themselves instantiate the fundamental physical properties, or whether (b) there are material objects located at the space-time points that instantiate these properties, or whether (c) there are no space-time points at all, the spatial-temporal relations being relations among material point particles that instantiate the fundamental physical properties.

What is crucial for this position is that the fundamental physical properties are intrinsic properties. According to the standard view developed by Lewis himself, intrinsic are all and only those properties that an object has irrespective of whether or not there are other contingent objects; in brief, having or lacking an intrinsic property is independent of accompaniment or loneliness (see Langton \& Lewis (1998) and for a refinement Lewis 
(2001)). All other properties are extrinsic or relational, consisting in the object bearing certain relations to other objects. The view hence is that the world is the distribution of fundamental physical intrinsic properties at points that are connected by spatio-temporal relations. This view is an atomism. The world consists of atoms in a philosophical sense, namely a plurality of objects that are characterized by certain intrinsic properties each and that are linked only by spatio-temporal relations.

There is an obvious epistemological problem for this position that is acknowledged by Lewis himself (see Lewis (forthcoming)): if the fundamental physical properties are intrinsic ones, how can we get knowledge of them? Frank Jackson brings out this problem in the following passage:

When physicists tell us about the properties they take to be fundamental, they tell us what these properties $d o$. This is no accident. We know about what things are like essentially through the way they impinge on us and our measuring instruments. It does not follow from this that the fundamental properties of current physics, or of 'completed' physics, are causal cum relational ones. It may be that our terms for the fundamental properties pick out the properties they do via the causal relations the properties enter into, but that at least some of the properties so picked out are intrinsic. They have, as we might put it, relational names but intrinsic essences. However, it does suggest ... the uncomfortable idea that we may know next to nothing about the intrinsic nature of the world. We know only its causal cum relational nature. (Jackson (1998), 23-24)

12 The core of this argument can be reconstructed as follows: (1) We gain empirical knowledge owing to the causal relations that obtain between physical objects and our senses. (2) Knowledge thus gained may refer to intrinsic properties of physical objects. (3) But the way in which that knowledge is caused imposes a constraint on its content: physical properties can be identified only through the relations in which they enter. If we explain the meaning of the propositions that refer to the fundamental physical properties, it turns out that these propositions describe these properties as relational. (4) Identity of relations, however, does not imply identity of intrinsic properties. (5) We therefore do not know the properties of physical objects insofar as they are intrinsic.

The argument is not that since we gain knowledge through the way in which physical objects impinge on our senses, we know only the way in which they are related to us. The argument is one about the content of empirical predicates, namely that they reveal only relations among objects. The argument applies to all relations; the relations in which physical objects stand to us do not have any special status as far as the content of empirical knowledge is concerned. The laws of physics, in short, describe relations among physical objects, and only relations, but without relations of measurement having a special status.

If it is true that our basic physical theories give us knowledge only of the relations in which physical objects stand, the metaphysics of intrinsic properties is in trouble: metaphysics has it that the world consists of objects that are characterized by intrinsic properties each. On epistemological reflection, however, we have to concede that we do not have access to these properties insofar as they are intrinsic. A gap between metaphysics and epistemology thus arises.

This problem for the metaphysics of intrinsic properties is a purely philosophical motivation to go for structural realism. One can reformulate the problem that Jackson among others raises in such a way that its conclusion is a position known as epistemic structural realism, namely the view that structure in the sense of relations among physical objects and 
as captured by the mathematical equations of a physical theory is all that we can know. Epistemic structural realism in the current discussion goes back to a paper that John Worrall published in 1989 (see in particular 117-123).

Worrall's aim is to employ epistemic structural realism as an argument to establish a mitigated version of scientific realism. According to him, there is continuity in our views about structure despite theory change in the history of science: the views about structure of a predecessor theory 13 can be construed as an approximation of the views about structure of the successor theory. Therefore, following Worrall, our views about structure do not fall victim to the arguments against scientific realism from theory change. That claim is in dispute (see Psillos (1999), chapter 7, against Worrall). Be that as it may, our concern in this paper is with the metaphysics of science. We shall therefore not employ structural realism as an argument for scientific realism, but simply presuppose that a version of scientific realism can be established that is strong enough to warrant the project of proposing metaphysical claims based on scientific theories. Of course, these claims then are subject to change in the same way as are our scientific theories.

Structural realism as a metaphysical position is the claim that there are no fundamental intrinsic properties underlying the relations that we can know. That is to say, all there is to the fundamental physical objects are the relations in which they stand. By structure, we mean concrete physical relations. Structural realism as a stance in the metaphysics of science is therefore not subject to what is known as the Newman objection against structuralism (see Demopoulos \& Friedman (1985); see Chakravartty (2004), section 3 as regards the point that concrete relations are not subject to this objection). As a first approximation, one can conceive structural realism as not touching the objects, but as replacing what is considered as intrinsic properties of objects in atomism with relations among the objects. However, this is only a first approximation, for it presupposes that there first are objects as something ontologically primitive and that these objects then are put into relations with each other ("first" and "then" in a logical sense, not a temporal one).

The structural realist, by contrast, maintains that objects and relations are on the same ontological footing. Neither objects nor relations (structure) have an ontological priority with respect to the physical world: they both belong to the ontological ground floor. It makes no sense to assign an ontological priority to objects, because instead of having fundamental intrinsic properties, there are only the relations in which they stand. In other words, an object as such is nothing but that what bears the relations. As regards the relations, it makes no sense to attribute an ontological priority to them, for at least insofar as they are instantiated in the physical world instead of being abstract entities, they exist as relations between objects. Thus, as far as the physical world is concerned, there is a mutual ontological as well as conceptual dependence between objects and structure (relations): objects can neither exist nor be conceived without relations in which they stand, and relations can neither exist in the physical world nor 14 be conceived as the structure of the physical world without objects that stand in the relations.

Structural realism is a holism in contrast to an atomism. In atomism, one considers the world as being composed of atoms in the sense of objects that exist independently of one another because they are characterized by intrinsic properties each ("local matters of particular fact, just one little thing and then another", in the words of David Lewis in the citation above). Holism, by contrast, can be conceived as regarding the whole world - or the 
domain of the world that one considers - as just one object in the last resort. "Object" here has the same meaning as in atomism, namely "being that exists independently of other beings" (this is one sense of the traditional term "substance"). All the properties of that one object trivially are intrinsic properties, for there is nothing outside that object. Structural realism rejects only the view of intrinsic properties underlying the relations, not intrinsic properties of a whole that is the only object in the last resort. It is trivial that any relations among the parts of a whole can be represented as intrinsic properties of the whole (although the converse is not the case). However, holism would collapse into atomism if the one whole did not have an internal structure. The claim would then simply be that there is just one atom.

The notion of an internal structure of the whole is therefore central to holism (see Esfeld (1998)). The idea is that there is an internal differentiation within the whole such that there are parts of the whole, and these parts have relational properties, that is, they stand in certain relations to one another. The parts are objects in a weaker sense than the whole - or an atom is: they do not exist independently of one another, but they are subjects of the predication of properties, namely relational properties, standing in relations. In the following, we shall talk about the parts of the whole as objects. When we talk about the whole, we explicitly mention this.

The idea hence is that the objects that are parts of the whole have only relational properties and no intrinsic ones. In other words, they are nothing but that what stands in the relations. This idea may seem incoherent. There is a master argument for intrinsic properties that can be put in this way:

1) Relations require relata, that is, objects that stand in the relations.

2) These objects have to be something in themselves, that is, they necessarily have some intrinsic properties over and above the relations that they bear to one another - even if the relations do not 15 supervene on the intrinsic properties and even if we cannot know the intrinsic properties (see, for instance, Langton (1998), chapter 2, in particular p. 22, who attributes that argument to Kant).

Structural realism rejects that argument. More precisely, the position that we shall put forward under the name of moderate structural realism accepts the first claim of this argument, but refuses to endorse the second one (see Esfeld (2004), section 3, and Esfeld \& Lam (2006), section 1). Whereas the first claim of this argument can be considered as a conceptual truth ("no relations without relata"), the second claim is clearly not a conceptual truth. It is rather a prejudice based on simply presupposing atomism. There are strong empirical arguments stemming from quantum physics and general relativity against that claim. We shall present these arguments in the second and the third section of this paper.

However, one may wonder whether relations are capable of individuating objects. If there are objects, don't they require intrinsic properties as identity condition? Recall that, according to structural realism as a metaphysical position, (1) objects are not atoms that exist independently of each other and that (2) structure always consists in certain specific, concrete relations, these relations being as determinate as intrinsic properties are supposed to be. Consequently, relations are exactly on the same footing as intrinsic properties as far as identity conditions are concerned: insofar as intrinsic properties account for identity conditions, relations can perform that task as well. For instance, if $A$ is bigger than $B$, heavier than $C$, etc., these relations individuate $A$ and distinguish $A$ from $B$ and $C$. It goes without saying that there is in structural realism no question of identity conditions for an object 
independently of other objects. But this does not mean that relations cannot provide identity conditions. Which relations make up for identity conditions for which types of objects depends obviously on the case under consideration.

Consider an analogy: since Quine's seminal paper on “Two dogmas of empiricism" (1951) and the subsequent development of semantic holism (inferential role semantics), we are familiar with the notion of a web of beliefs. We are used to thinking of beliefs as points in a web that are individuated by their position in the web, that is, their relations to other beliefs. Content (meaning) is not an intrinsic property of a belief, but consists in inferential relations to other beliefs (the same goes for other properties of beliefs such as confirmation or justification). Semantic holism has no problem in individuating beliefs on that basis: each belief is defined by its position in 16 the web, being distinguished from all the other beliefs in the web, for no two beliefs stand in exactly the same relations to all the other beliefs in the web. The problem is that we do not want any old change of relations in the system to amount to a change in the content of all the beliefs in the system. Some inferential relations thus have to be distinguished as being more important than others. But this problem does not touch the central issue that it is relations that provide the identity conditions for the members of the system. Structural realism can be received as proposing to transfer this idea from semantics to metaphysics, the objects being now physical entities instead of beliefs. If this idea is intelligible in semantics, then so it is in metaphysics.

Hence, insofar as intrinsic properties can provide identity conditions, so can relations. However, there are cases in physics where neither relations nor intrinsic properties are able to establish identity conditions. Quantum systems of the same kind whose states are entangled are indistinguishable (see French \& Redhead (1988)), although in the common cases there is a definite number of them that is greater than one. These systems do not have an identity in time. An analogous consideration applies to space-time points on certain symmetry assumptions about space-time: space-time points can stand in exactly the same spatiotemporal relations and, yet, be of course numerically distinct (see below section 3).

One may receive these cases as speaking against a bundle theory of objects: quantum systems and space-time points can neither be bundles of intrinsic properties nor can they be bundles of relational properties; for the intrinsic or relational properties may be as concrete as is physically possible and, nevertheless, fail to establish a distinction between quantum systems or space-time points. A bundle theory of objects accords ontological priority to intrinsic properties or relations over objects: objects are constituted by intrinsic properties or relations on that theory.

The other big position in the metaphysics of objects apart from the bundle theory is the view that objects are bare particulars: each object has a primitive thisness (haecceity). It is that primitive thisness which individuates the object and provides its identity conditions (see Adams (1979)). Primitive thisness is not a property. It functions rather like a proper name. If there are one hundred entries under the name "Jones" in a telephone directory, this does not mean that there are one hundred instantiations of the property of being Jones in the space-time region to which the telephone directory applies. However, as far as quantum systems are concerned, one can complain that primitive thisness is a purely metaphysical position for which 17 there cannot be any empirical argument stemming from science. More importantly, as far as space-time points are concerned, there is a strong argument against primitive thisness on which we shall elaborate in section 3, namely the hole argument. The view of each object 
having a primitive thisness accords ontological priority to objects over intrinsic properties or relations: objects are first constituted by a primitive thisness that provides for their identity and then equipped with intrinsic properties or put into relations ("first" and "then" in a logical sense, not a temporal one). The view of objects being constituted by a primitive thisness stands in opposition to the spirit of structural realism.

The bundle theory and the view of objects as bare particulars are not the only options in the metaphysics of objects. In the cases where neither intrinsic properties nor relations provide for identity conditions one can simply accept a numerical distinction (diversity) - among quantum systems or space-time points - as primitive (a similar view is held by Pooley (2006), section 4). A numerical distinction tells us that there is a number of objects that is greater than one - in many cases of quantum entanglement even a definite, finite natural number of objects -, and that is all that it tells us. A numerical distinction is not a primitive thisness, for it does not establish an identity in time - or any other sort of an identity - that is not empirically accessible. Accepting a numerical distinction as primitive is motivated by the physical cases - quantum entanglement, space-time points - in which there is a plurality of objects without these objects being distinguished from one another by any intrinsic properties or relations in which they stand and without primitive thisness being an open way out, since there are strong physical arguments against primitive thisness. This empirical situation - and thus the motivation for acknowledging numerical distinction as a primitive - is independent of structural realism. Any position in the metaphysics of science has to come to terms with this empirical situation.

Nonetheless, recognizing numerical distinction as a primitive is the reason why we are committed to the view that objects and relations are interdependent, being on the same ontological footing: we get the relata and the relations at once as the internal structure of a whole, neither of them being eliminable or reducible to the other one. Hence, in short, insofar as there are factors that individuate objects over and above numerical distinction, intrinsic properties and relations are on a par. If there are no such factors, we either have to accept a numerical distinction as primitive or we have to go for primitive thisness. Moderate structural realism is committed to the former view.

18 As the characterization as moderate suggests, there is a more radical version of structural realism. The view that Steven French and James Ladyman put forward under the name "ontic structural realism" is a radical metaphysics of structural realism in contrast to the more moderate position proposed by us, because it rejects both claims of the above mentioned master arguments for intrinsic properties. According to French and Ladyman, the fact that quantum systems of the same kind in entangled states are indistinguishable is a good reason to drop the commitment to objects in metaphysics. They maintain a metaphysics of structural realism according to which there is only structure in the sense of concrete, physical relations, but no objects standing in the relations, the objects being dissolved into structure. Their view, however, has become less radical recently, since they seem to be prepared now to admit objects as a secondary category, being derived from relations (see Ladyman (1998), French \& Ladyman (2003) for the original view and Ladyman \& Ross (2007), chapter 3, in particular the opening paragraph, as well as French (2006), section 3, in particular first paragraph on p. 6 for more recent, less radical statements. For another version of a radical structural realism independent of the one of French \& Ladyman see Dipert (1997)). 
Be that as it may, if objects are conceived as being derived from relations, one would like to see how this can be so, given that relations fail to distinguish between objects in the cases of quantum entanglement and space-time points. And if there are no objects at all, the complaint is that structural realism runs into absurdity: for the relations to be instantiated in the physical world, there has to be something that instantiates them, that is, something that stands in the relations. That is why the first claim of the master argument for intrinsic properties is a conceptual truth if anything is (as to this objection against the position of French \& Ladyman, see Cao (2003b), Chakravartty (2003), 871-872, Busch (2003), Psillos (2005), section 2). And that is why the version of structural realism that we endorse puts objects on the same ontological footing as relations. The difference between the radical structural realism of French and Ladyman and our more moderate version is, however, a difference in detail within a common position. The central element of structural realism as a position in the metaphysics of science is the commitment to relations instead of intrinsic properties and the rejection of an atomistic in favour of a holistic metaphysics consequent upon that commitment.

One can put forward three types of arguments for structural realism as a stance in the metaphysics of science:

- 19 the argument from coherence: Our metaphysics should be coherent with our epistemology. Postulating intrinsic properties that are unknowable leads to a gap between metaphysics and epistemology as explained above. Structural realism makes metaphysics coherent with epistemology: all there is can in principle be known; for all there is are relations among objects. There is no principled obstacle to the knowledge of relations, whereas there is a principled obstacle to the knowledge of intrinsic properties (although all our physical theories are, of course, at best approximately true).

- the argument from parsimony: We have to recognize relations (structure) in our metaphysics anyway. It is not possible to reduce all relations to intrinsic properties. Even if, as according to atomism, the world consists of objects whose fundamental properties are intrinsic ones, there have to be some relations: at least spatio-temporal relations are not supervenient on - and consequently not reducible to - intrinsic properties (that much is conceded even by David Lewis in his thesis of Humean supervenience; see the quotation at the beginning of the paper). On the other hand, it is questionable whether we have to recognize both relations and intrinsic properties in our metaphysics. Parsimony (Occam's razor) tells us that we shall not admit entities beyond necessity. Thus, the claim is that the metaphysics of structural realism is parsimonious, because it does not recognize more than is necessary anyway, namely relations (structure).

- the empirical arguments from quantum entanglement and space-time: The argument from coherence is a general argument that applies to all our knowledge of the physical world, physics be as it may, saying that there is no reason to suppose that there are fundamental intrinsic properties. There are two concrete arguments based on our current two fundamental physical theories that establish a stronger conclusion: the assumption that there are fundamental intrinsic properties underlying the relations leads to a conflict with what these theories tell us about the physical world.

Before turning to these arguments and to conclude this section, let us come back to the contrast between structural realism and David Lewis' thesis of Humean metaphysics quoted at the beginning of the paper. A world to which the metaphysics of structural realism applies 
is a world of holism, being tied together by relations that do not supervene on intrinsic 20 properties - by contrast to a world of atomism that is composed of objects that exist independently of one another, being characterized by intrinsic properties each. However, holism is not committed to the anti-Humean thesis that there are necessary connections among distinct entities in the world. The distribution of relations in a world of structural realism can be contingent in the same way as the distribution of intrinsic properties in David Lewis' Hume world.

More precisely, the relations that there are in one part of the world do not have to necessitate the relations that there are beyond the part of the world considered. To illustrate this claim by means of an example from non-relativistic quantum mechanics, assume that there is a state of the world which is an entangled state and that this state develops in time. Structural realism is compatible with the view that the state of the world at a given time does not necessitate the state of the world at other times. Thus, structural realism is compatible with the view that there are no necessary connections among the state of the world at different times. The dynamics of the development of the state of the world in time may of course be deterministic (such as the Schrödinger equation). But a Hume world can be deterministic too. Physical determinism does not imply the view that there are metaphysically necessary connections in the world.

Consequently, in structural realism as in a Hume world, the laws of nature can be contingent instead of being metaphysically necessary. The issue of contingency vs. metaphysical necessity is independent of the issue of intrinsic properties vs. relations. Structural realism and holism can go with both of these views - in the same way as one can combine a metaphysics of intrinsic properties with a Humean world view as well as with the view that there are metaphysically necessary connections in the world (for instance, by conceiving the fundamental intrinsic properties as powers).

\section{The argument from quantum entanglement}

If we try to translate David Lewis' thesis of Humean supervenience into physics, we can make use of the principle of separability. Einstein based his criticism of quantum mechanics on this principle (see Einstein (1948) and Howard (1985)). One can characterize separability in this way: Physical systems have a state each in the sense that (1) this state completely encompasses the state-dependent properties of the system and (2) the joint state of two or more systems supervenes on the states which each of these systems has. Physical systems may be particles, field modes, space-time points, etc. In non-relativistic 21 quantum mechanics, the state of a system at a time can be conceived as containing the complete information about the properties of the system at that time, the properties being limited to those properties whose value can change in time. These are known as state-dependent properties. Properties such as rest mass and charge, by contrast, are state-independent, since their value always remains constant. The principle of separability thus conceives the world as being built up of single systems each of which has a state independently of all the other systems, and the joint state of two or more systems - or, in the last resort, the whole world supervenes on the states that these systems have independently of each other. In other words, the relations among the systems supervene on the states that the systems have independently of each other; consequently, the state-dependent properties are conceived as intrinsic properties. 
Quantum entanglement violates separability. If the states of two or more quantum systems are entangled, only the joint state of the whole is a pure state. The parts, the single systems whose states are entangled, do not have a state each that completely encompasses their statedependent properties. Instead of the parts fixing the state of the whole, it is only the joint state of the whole that completely determines the state-dependent properties of the parts in the form of certain correlations among these properties, entanglement signifying that there is a superposition of all the possible correlations. This way of determining the properties of the parts in the form of correlations among them makes it superfluous to call for intrinsic properties underlying the correlations. Claiming that there are intrinsic and thus local properties of the parts that serve as a supervenience base for the correlations would come into conflict with the fact that the correlations of quantum entanglement violate the theorem of Bell (1964) (as regards the philosophical importance of that theorem, see e.g. the papers in Cushing \& McMullin (1989)). Quantum mechanics hence is not silent on the issue of whether or not there may be intrinsic properties underlying the correlations, but contains a strong argument against any such view.

Quantum non-separability fits into structural realism as sketched out in the first section of this paper (for a detailed argument in this sense, see Esfeld (2004); see furthermore Teller (1986), Howard (1989), Healey (1991) on the link with holism and Ladyman (1998) and French \& Ladyman (2003) on the link with structural realism). The way in which the joint state of the whole determines the state-dependent properties of the parts in the form of certain correlations confirms the claim of a mutual ontological dependence between objects and relations: the objects (single quantum systems - "particles" in the framework of nonrelativistic quantum mechanics) cannot 22 be presupposed as simply being there and then entering into correlations (for instance, through interaction). Quantum entanglement is generic and fundamental. We cannot but take as fundamental the joint state of the whole, in the last resort the joint state of the whole world. That state is such that it permits and calls for an internal differentiation in the form of correlations and thus correlata - although the correlata are nothing but that what stands in the correlations. We thus get correlations and correlata as internal differentiation of the world, these two being on the same ontological footing.

The interpretation of quantum entanglement in terms of holism and structural realism is independent of the stance that one takes on the measurement problem. If one follows Everett (1957) in holding that the Schrödinger equation is the complete dynamics of quantum systems, there only is quantum entanglement. If one modifies the Schrödinger dynamics - as, for instance, along the lines of the proposal of Ghirardi, Rimini \& Weber (1986) - to allow for state reductions and thus processes of the dissolution of quantum entanglement, nonetheless, quantum entanglement is fundamental. To the extent that there are pure states of single quantum systems, they are derived from entanglement.

The argument from quantum entanglement in favour of structural realism can be considered in a general framework: it takes the physical relation of quantum entanglement as a fundamental feature of the world - whatever the fundamental objects standing in the relation are. In particular, the argument is not restricted to non-relativistic quantum theory. It applies in the framework of (relativistic) quantum field theory (QFT) as well, according to which entanglement is a fundamental feature of nature too (see Clifton \& Halvorson (2001)). In other words, the structural realist interpretation is not tied to any particular ontology of basic 
entities; as regards QFT, quantum field systems can play the role of objects among which the relations of quantum entanglement obtain (where a quantum field system can be understood basically as a specified bounded open region of space-time in which some field properties are instantiated). Therefore, the argument from quantum entanglement for structural realism is independent of the "particle ontology vs. field ontology" debate in the philosophy of quantum field theory (in favour of a field ontology, see Huggett (2000) and Cao (2003a) for instance; for a more cautious stance based on some physical considerations, see Halvorson (forthcoming), sections 4 and 6).

The important point is that the relation of quantum entanglement is fundamental within quantum theory, for both relativistic and non-relativistic cases - fundamental in the sense of being non-supervenient upon intrinsic 23 properties and of involving non-separability. To the extent that quantum theory is our best physical theory about matter, this constitutes a strong empirical argument against the traditional atomist metaphysics of intrinsic properties and in favour of the structural realist account proposed in this paper, at least as far as matter is concerned. However, one may still wonder whether such a structural realist account provides a coherent metaphysics of space-time that accords with our contemporary fundamental physical description of it. Therefore, we now turn to the case of space-time as described by the theory of general relativity (GR).

\section{The argument from space-time}

At first sight and in a realist (substantivalist) move, it seems that the standard mathematical representation of space-time within contemporary physics in terms of a set of points endowed with certain topological, smooth differential and metric properties - the standard manifold description of space-time - constitutes a straightforward implementation of atomistic metaphysics such as David Lewis' thesis of Humean supervenience. Indeed, such representation of space-time seems to fit well into the conception of the world as a distribution of fundamental intrinsic properties (together with space-time relations) instantiated at space-time points or events, which then play the role of atoms in the philosophical sense. Whereas such a conception fares well with the non-general-relativistic representation of space-time as a fixed background, it, however, faces some serious difficulties within GR.

In this framework and in the standard formulation of the theory, space-time is represented by a four-dimensional smooth differentiable manifold - the above mentioned set of points with topological and smooth differential properties - together with a Lorentz metric tensor field, or metric for short, defined on it. This latter geometric object encodes the fundamental space-time relations, like the chronogeometrical relations (space-time intervals), the inertiogravitational relations (describing the behaviour of freely falling test particles in a gravitational field - the metric field and the gravitational field being one and the same field within GR) and the causal relations (defining a light cone at each space-time point and providing a distinction between spatial and temporal directions). One of the major novelties of GR is that the metric, incorporating the fundamental relations of the space-time structure, is fundamentally dynamical: it is related to the behaviour of the (non-gravitational) energymatter ("ordinary" 24 energy-matter), represented by the stress-energy tensor field, through the (non-linear) dynamical equations - the Einstein field equations - it satisfies. 
In this section, we mainly consider the pure gravitational cases, that is, the physical solutions of the Einstein field equations with vanishing non-gravitational stress-energy tensor (roughly, they are descriptions of space-time without "ordinary" energy-matter). This does not alter the main argument in favour of the metaphysical thesis of structural realism, nor does it make this argument dependent on a specific position with respect to the ontological status of space-time. We briefly discuss at the end of this section the link with this longstanding debate.

The important feature of GR for our considerations is the principle of active general covariance. This principle tells us that if we have a space-time model of GR, that is, a solution of the Einstein field equations, then any active diffeomorphism applied on this model will generate a space-time model of GR. An active diffeomorphism is a differentiable, one-to-one and onto mapping (with differentiable inverse) acting on the Lorentz metric and stress-energy tensor fields defined on the manifold. Such diffeomorphic models are observationally indistinguishable. However, in a substantivalist move and according to the traditional metaphysics of individuals applied to space-time, these diffeomorphic models have to be interpreted as describing distinct physical situations, since any given space-time point or event (merely represented by a manifold point from this perspective) is individuated by some intrinsic properties independently of the space-time relations represented by the metric. It will therefore be "coloured" by different metrical properties in the different diffeomorphic models. For instance, the question whether the metric (or gravitational) field is flat around some specific space-time point may receive different answers in the different diffeomorphic models.

The famous hole argument, originally due to Einstein, shows that such an attitude towards diffeomorphic models leads to a kind of indeterminism (see Earman \& Norton (1987)): we consider a hole in the space-time manifold, that is, an open subset of the manifold where all non-gravitational fields vanish. We furthermore consider a non-trivial active diffeomorphism on the hole that smoothly reduces to the trivial diffeomorphism, that is, the identity, on the boundary and outside the hole. A complete physical model outside the hole is then insufficient to determine a unique physical solution inside the hole, since, within the substantivalist and atomistic metaphysical framework, diffeomorphic models represent distinct situations. More 25 precisely, considering a space-time manifold that can be foliated, the "hole" can be chosen to be the portion of the manifold after a certain time $t$ in the considered foliation. But then, two physically possible models, which are related by a 'hole diffeomorphism' and in which we consider the same foliation, may agree till a time $t$ and then disagree for any time $t^{\prime}>t$ in the foliation. This constitutes a breakdown of common determinism, and no unique evolution can be determined from a set of initial data (in the initial value formulation of the theory).

Since diffeomorphic models are observationally indistinguishable, it is then generally argued that such indeterminism is not an empirically supported feature of the physical theory, but rather an artifact of the (metaphysical) conception of space-time that implies the physical non-equivalence of diffeomorphic models. Indeed, a wide range of philosophers of physics and physicists agree on the fact that this non-equivalence and the hole argument itself are a consequence of the non-physical primary individuation of space-time points independently of the metric (see for instance Stachel (1993), Brighouse (1994), Hoefer (1996), Dorato (2000), Pooley (forthcoming)). In other words, space-time points are not individuals independently of 
the space-time relations they enter into, which are represented by the metric; they do not possess any primitive thisness (haecceity) or intrinsic properties that would turn them into individuals over and above bearing the space-time relations. Therefore, with respect to spacetime, the fundamental GR-principle of active general covariance, which underlies the hole argument, constitutes a strong empirical argument against the traditional atomistic metaphysics of individuals. On the contrary, the (holistic) metaphysical framework of structural realism provides a convincing and coherent account of the physical description of space-time provided by GR. Indeed, with respect to active general covariance and the GRrepresentation of space-time in terms of a manifold with a dynamical metric that encodes all the fundamental space-time relations, space-time can be naturally understood as a purely relational physical structure, that is, a network of space-time relations among space-time points that do not possess any intrinsic properties.

Moreover, the space-time structure described by GR is such that the space-time relations and the objects that stand in the relations (the space-time points or events) are on the same (fundamental) ontological footing. On the one hand and in an analogous way to the general case discussed in the first section, it makes no sense to consider an actual (that is, instantiated in the physical world) space-time relation without relata standing in the relation - space-time points or events in the pure gravitational cases. 26 On the other hand, the physical description of space-time within GR (and in particular the principle of active general covariance) makes meaningless any individuation of space-time points (with the help of intrinsic properties or of primitive thisness for instance) independently of the space-time relations they inter into or independently of the space-time structure they are part of - both being represented by the metric. Space-time points do not possess any independent existence (they are not atoms in the philosophical sense), but only exist in virtue of their standing in relation to other space-time points. There is no ontological priority, but rather a mutual ontological dependence between space-time relations and space-time points.

As regards individuation and identity conditions for space-time points, we argue, as explained in the first section, that space-time relations, which are concrete and determinate relations, are on a par with intrinsic properties. In a space-time with no symmetries, a spacetime point can be individuated, at least in principle, through the unique way it stands in (space-time) relations to other space-time points. A concrete physical implementation of such individuation within GR is the Bergmann-Komar assignment to space-time points of four scalar polynomials of the curvature, where the curvature can be understood as a functional of the metric (for recent developments, see Lusanna \& Pauri (2004)). What about the cases with symmetries, like for instance the homogeneous and isotropic Friedman-Lemaître-RobertsonWalker solutions, which constitute the 'standard model' of contemporary cosmology? In these cases, the above mentioned individuation procedure becomes degenerated and, in general, no properties - intrinsic or relational - seem to be able to provide well-defined identity conditions for space-time points. However, this is not a difficulty for the structural conception of space-time points (and of objects in general, see section 1) proposed here, since in this metaphysical framework, space-time points and space-time relations are on the same ontological footing: the numerical distinction of space-time points is neither reduced to spacetime relations nor grounded independently of them (by some intrinsic properties or primitive thisness). Therefore, the numerical distinction of space-time points can be accepted as primitive in the same way as space-time points and space-time relations - the whole space- 
time structure indeed - can. Thus, within the structural realist interpretation of space-time, even in the cases with symmetries, there is a metaphysically coherent notion of numerical distinction for space-time points. As a physical consequence of this kind of 'structural individuality' implied by the theory, the space-time location of any physical entity (like 'being localized at a space-time point or in a space-time 27 region') makes only sense within GR with respect to the dynamical space-time structure, that is, with respect to the metric (or gravitational) field (see Rovelli (2004), chapter 2).

The main claim of this section is that there are strong empirical arguments from contemporary space-time physics against the traditional atomistic understanding of spacetime in terms of a set of individuals possessing intrinsic properties (together with some spacetime relations). We have seen that the holistic metaphysics of structural realism provides a convincing and coherent account of space-time as described by GR. According to this view, space-time is rather a network of space-time relations among constituents (space-time points) that do not possess intrinsic properties. However, this metaphysical claim about space-time does not constitute a clear-cut position in the debates about the ontological status of spacetime and about the relationship between space-time and (non-gravitational) energy-matter. In particular, the structural realist conception of space-time is open with respect to whether or not the space-time structure and (non-gravitational) energy-matter are distinct ontological beings. If the space-time structure can be ontologically dependent on there being some nongravitational energy-matter, we want to stress that structural realism about space-time has nothing to do with any kind of relationalism about space-time understood in the reductive sense, since the space-time structure is not reduced to something non-spatio-temporal. On the contrary, the structural realist thesis about space-time, claiming the existence of the spacetime structure, seems to be committed to some kind of substantivalism with respect to spacetime. But it leaves the question of the relationship between space-time and non-gravitational energy-matter open - substantivalism à la Newton (matter ontologically distinct from spacetime) or substantivalism à la Spinoza (matter identical with space-time).

\section{4. $\quad$ Conclusion and open issues}

In the first section of this paper, we have set out structural realism as an ontological thesis about the world. The main claim of this postion is that the physical world is at the fundamental level a purely relational structure, that is, a network of relations among objects that do not possess intrinsic properties. The version of structural realism that we propose is a moderate one, since it regards relations and objects as being mutually ontologically dependent. This position implies that there are no fundamental intrinsic propreties in nature. It thereby avoids a gap between what we can know about the world and what the world really consists of.

28 We have argued that this structural realist position is coherent, answering the objections usually raised against structuralism. The Newman objection against formal structures has no force in the case of the actual concrete relations considered by moderate structural realism. Moreover, the metaphysics of objects provided by the moderate structural realist position rejects any commitment to haecceitism, but it does not amount to the bundle theory of objects. Since structure is conceived in such a way that objects and relations are ontologically on a par, a numerical distinction among the objects can be considered as primitive in the sense of being already incorporated into the notion of a concrete, physical structure. 
We have not only shown that the metaphysical thesis of moderate structural realism is coherent, we have also argued in section 2 and 3 that it is strongly supported by empirical arguments from our fundamental physical theories. Both GR and Q(F)T describe fundamental physical relations - namely, the quantm entanglement relations and the space-time relations that are irreducible to and non-supervenient on intrinsic properties of the physical relata standing in the relations. These physical relations belong therefore to the most fundamental part of the physical descriptions of the world. In a scientific realist move, they have to be recognized in any metaphysical framework that pays heed to contemporary physics. Nonetheless, some work remains to be done to justify the conclusion that all the fundamental physical features of nature are best understood in this holistic metaphysical framework. For instance, state-independent fundamental features of quantum (field) systems, such as (rest) mass, charge, spin, etc. seem to be good candidates for intrinsic properties (they are, however, of no help in individuating entangled quantum (field) systems). A possible line of investigation for the structural realist is to look at the mathematical (namely, group-theoretic) structures in terms of which these properties are defined and which therefore may have some physical significance.

The mutual ontological dependence between relations and objects proposed by moderate structural realism holds whatever the relations are (quantum, spatio-temporal) and whatever the objects are (single quantum systems, space-time points). However, it is committed to there being some fundamental objects standing in the relations - there is no infinite regress (no 'structures all the way down'). Ultimately, the structural realist's understanding of nature is therefore open with respect to what kind of fundamental objects and relations there are in the world, as long as their relationship is conceived as one of mutual ontological dependence. We therefore contend that this central idea of moderate structural realism 29 will continue to prove sound even if current quantum field theory and general relativity will be replaced with a new fundamental physical theory that offers a unified view of matter and space-time.

\section{References}

Adams, Robert M. (1979): "Primitive thisness and primitive identity”. Journal of Philosophy 76, pp. 5-26.

Bell, John S. (1964): “On the Einstein-Podolsky-Rosen-paradox”. Physics 1, pp. 195-200.

Brighouse, Carolyn (1994): “Spacetime and holes”. In D. Hull, M. Forbes \& R.M. Burian (eds.): PSA 1994. Proceedings of the 1994 biennial meeting of the Philosophy of Science Association. Volume 1. East Lansing: Philosophy of Science Association. Pp. 117-125.

Busch, Jacob (2003): "What structures could not be". International Studies in the Philosophy of Science 17, pp. 211-223.

Cao, Tian Yu (2003a): "Structural realism and the interpretation of quantum field theory". Synthese 136, pp. $3-24$.

Cao, Tian Yu (2003b): “Can we dissolve physical entities into mathematical structure?”. Synthese 136, pp. 51-71.

Chakravartty, Anjan (2003): “The structuralist conception of objects”. Philosophy of Science 70, pp. 867-878.

Chakravartty, Anjan (2004): "Structuralism as a form of scientific realism". International Studies in the Philosophy of Science 18, pp. 151-171.

Clifton, Rob \& Halvorson, Hans (2001):“Entanglement and open systems in algebraic quantum field theory”. Studies in History and Philosophy of Modern Physics 32, pp. 1-31.

Cushing, James T. \& McMullin, Ernan (eds.) (1989): Philosophical consequences of quantum theory. Reflections on Bell's theorem. Notre Dame: University of Notre Dame Press. 
Demopoulos, William \& Friedman, Michael (1985): “Critical notice: Bertrand Russell's The analysis of matter: its historical context and contemporary interest". Philosophy of Science 52, pp. 621-639.

Dipert, Randall R. (1997): "The mathematical structure of the world: the world as a graph". Journal of Philosophy 94, pp. 329-358.

Dorato, Mauro (2000): "Substantivalism, relationism, and structural spacetime realism". Foundations of Physics 30 , pp. $1605-1628$.

Earman, John \& Norton, John (1987): "What price spacetime substantivalism? The hole story". British Journal for the Philosophy of Science 38, pp. 515-525.

Einstein, Albert (1948): "Quanten-Mechanik und Wirklichkeit”. Dialectica 2, pp. 320-324.

Esfeld, Michael (1998): "Holism and analytic philosophy". Mind 107, pp. 365-380.

Esfeld, Michael (2004): "Quantum entanglement and a metaphysics of relations". Studies in History and Philosophy of Modern Physics 35B, pp. 601-617.

Esfeld, Michael \& Lam, Vincent (2006): "Moderate structural realism about space-time". Forthcoming in Synthese, online prepublication Synthese online http://springerlink.metapress.com/content/1573-0964. Also available on http://philsci-archive.pitt.edu/archive/00002778/

Everett, Hugh (1957): “Relative state' formulation of quantum mechanics”. Reviews of Modern Physics 29, pp. 454-462. Reprinted in B. S. DeWitt \& N. Graham (eds.) (1973): The many-worlds interpretation of quantum mechanics. Princeton: Princeton University Press. Pp. 141-149.

French, Steven (2006): "Identity and individuality in quantum theory". In: E. N. Zalta (ed.): The Stanford Encyclopedia of Philosophy. http://plato.stanford.edu/archives/spr2006/entries/qt-idind/

French, Steven \& Ladyman, James (2003): "Remodelling structural realism: quantum physics and the metaphysics of structure". Synthese 136, pp. 31-56.

French, Steven \& Redhead, Michael L. G. (1988): "Quantum physics and the identity of indiscernibles". British Journal for the Philosophy of Science 39, pp. 233-246.

Ghirardi, Giancarlo, Rimini, Alberto \& Weber, Tullio (1986): "Unified dynamics for microscopic and macroscopic systems". Physical Review D34, pp. 470-491.

Halvorson, Hans (forthcoming): “Algebraic quantum field theory”. In: J.Butterfield \& J.Earman (eds.): Handbook for the Philosophy of Physics. Elsevier.

Healey, Richard A. (1991): "Holism and nonseparability". Journal of Philosophy 88, pp. 393-421.

Hoefer, Carl (1996): "The metaphysics of space-time substantivalism”. Journal of Philosophy 93, pp. 5-27.

Howard, Don (1985): "Einstein on locality and separability". Studies in History and Philosophy of Science 16, pp. 171-201.

Howard, Don (1989): "Holism, separability, and the metaphysical implications of the Bell experiments". In: J. T. Cushing \& E. McMullin (eds.): Philosophical consequences of quantum theory. Reflections on Bell's theorem. Notre Dame: University of Notre Dame Press. Pp. 224-253.

Huggett, Nick (2000): "Philosophical foundations of quantum field theory". British Journal for the Philosophy of Science 51, pp. 617-637.

Jackson, Frank (1998): From metaphysics to ethics. A defence of conceptual analysis. Oxford: Oxford University Press.

Ladyman, James (1998): "What is structural realism?" Studies in History and Philosophy of Modern Science 29, pp. $409-424$.

Ladyman, James \& Ross, Don with Spurrett, David \& Collier, John (2007): Every thing must go. Metaphysics naturalised. Oxford: Oxford University Press.

Langton, Rae (1998): Kantian humility. Our ignorance of things in themselves. Oxford: Oxford University Press.

Langton, Rae \& Lewis, David (1998): "Defining 'intrinsic"'. Philosophy and Phenomenological Research 58, pp. 333-345.

Lewis, David (1986): Philosophical papers. Volume 2. Oxford: Oxford University Press.

Lewis, David (2001): "Redefining 'intrinsic"'. Philosophy and Phenomenological Research 63, pp. 381-398.

Lewis, David (forthcoming): "Ramseyan humility". Forthcoming in D. Braddon-Mitchell, R. Nola \& D. Lewis (eds.): The Canberra programme. Oxford: Oxford University Press. 
Lusanna, Luca \& Pauri, Massimo (2004): "General covariance and the objectivity of space-time point-events". Invited Contribution to the ESF 2004 Oxford Conference on Space-Time. arXiv: gr-qc/0503069.

Pooley, Oliver (2006): "Points, particles, and structural realism". In: S. French, D. Rickles and J. Saatsi (eds.): Structural foundations of quantum gravity. Oxford: Oxford University Press. Pp. 83-120.

Psillos, Stathis (1999): Scientific realism. How science tracks truth. London : Routledge.

Psillos, Stathis (2006): "The structure, the whole structure and nothing but the structure". Philosophy of Science 73 (Proceedings), pp. 560-570.

Quine, Willard Van Orman (1951): “Two dogmas of empiricism”. Philosophical Review 60, pp. 20-43.

Rovelli, Carlo (2004): Quantum gravity. Cambridge: Cambridge University Press.

Stachel, John (1993) : "The meaning of general covariance. The hole story". In: J. Earman, I. Janis, G. J. Massey $\&$ N. Rescher (eds.): Philosophical problems of the internal and external worlds. Essays on the philosophy of Adolf Gruenbaum. Pittsburgh: University of Pittsburgh Press. Pp. 129-160.

Teller, Paul (1986): "Relational holism and quantum mechanics". British Journal for the Philosophy of Science 37, pp. 71-81.

Worrall, John (1989): "Structural realism: the best of two worlds?" Dialectica 43, pp. 99-124. Reprinted in D. Papineau (ed.) (1996): The philosophy of science. Oxford: Oxford University Press. Pp. 139-165. 